

А. А. Мастихина, Критерий частичного предвосхищения общерегулярных сверхсобытий, Дискрет. матем., 2011, том 23, выпуск 4, 103-114

DOI: https://doi.org/10.4213/dm1164

Использование Общероссийского математического портала Math-Net.Ru подразумевает, что вы прочитали и согласны с пользовательским соглашением http://www.mathnet.ru/rus/agreement

Параметры загрузки:

IP : 3.85 .5 .30

26 апреля 2023 г., $17: 34: 46$ 


\title{
Критерий частичного предвосхищения общерегулярных сверхсобытий
}

\author{
() 2011 г. А. А. Мастихина
}

\begin{abstract}
Автомат угадывает следующий символ входной последовательности, если он выдает этот символ на выходе в предыдущий момент времени. В работе рассмотрен вопрос, можно ли с помощью конечного автомата частично предвосхитить общерегулярное сверхсобытие, то есть угадать некоторую ненулевую долю символов каждого сверхслова из этого сверхсобытия.
\end{abstract}

Получены критерий и алгоритм, позволяющие это установить.

\section{1. Введение}

В статье рассматривается предвосхищение сверхсобытий над алфавитом $\{0,1\}$ определенного вида с помощью конечных автоматов. Понятие автомата с предвосхищением возникло еще в начале развития теории автоматов [1].

Это означало, что автомат использует не только данные, уже поступившие на вход, но и некоторые значения, которые должны поступить в будущем. На практике в качестве еще не поступивших данных часто используется наиболее вероятное значение.

В данной работе рассматриваются автоматы, не использующие заведомо известную информацию, которая должна поступить на вход, а как раз пытающиеся эти данные предсказать на выходном канале.

В [2] рассматривались автоматы, детерминирующие сверхслово или множество сверхслов. Это значило, что через некоторое конечное время автомат начинает угадывать каждый следующий символ, то есть на выходе в момент времени $t$ выдавать элемент входной последовательности с номером $t+1$.

Было показано, что детерминируемо только множество периодических сверхслов с фиксированным периодом.

Поэтому было введено понятие частичного предвосхищения, в [3] называемое частичным угадыванием. Автомат верно предсказывает некоторую долю входных символов, которая называется степенью предвосхищения и определяется как нижний предел отношения числа угаданных символов за время $t$ к $t$ при $t$, устремленном к бесконечности. Там же было показано, что можно построить такое сверхслово, что для каждого автомата степень предвосхищения нулевая.

Здесь автомату подаются на вход сверхслова из некоторого фиксированного множества, и его цель - предсказать каждое сверхслово из этого множества хотя бы с ненулевой степенью. 
Рассматриваются общерегулярные сверхсобытия, то есть множества сверхслов, представимые в конечных автоматах. Получен критерий, позволяющий для любого общерегулярного сверхсобытия определить, существует ли для него автомат с частичным предвосхищением. Эта задача алгоритмически разрешима за линейное время от числа состояний минимального автомата, представляющего сверхсобытие. При положительном ответе соответствующий автомат строится.

Сверхсобытие может быть задано автоматом или общерегулярным выражением. В критерии используется структура автомата.

Сначала критерий получен для сверхитерации регулярного события, потом этот результат обобщен для произвольных общерегулярных сверхсобытий.

Автор выражает благодарность профессору Э. Э. Гасанову за постановку задачи и помощь в работе.

\section{2. Основные понятия и формулировка результатов}

Будем использовать следующие обозначения:

$A^{*}$ - множество всех слов в алфавите $A$, по определению будем считать, что пустое слово $\Lambda$ принадлежит $A^{*}$,

$A^{\infty}$ - множество всех сверхслов в алфавите $A$,

$|a|-$ длина слова $a \in A *$, по определению $|\Lambda|=0$,

$a(n)-n$-ый элемент слова или сверхслова $a$,

$\operatorname{pref}(a, n)-$ префикс слова или сверхслова $a$ длины $n$, то есть $\operatorname{pref}(a, n)=a(1) \ldots a(n)$,

$\operatorname{post}(\alpha, n)=\alpha(n+1) \alpha(n+2) \ldots-$ сверхслово или слово длины, не меньшей $n$, без первых $n$ символов,

событие - подмножество $A^{*} /\{\Lambda\}$,

сверхсобытие - подмножество $A^{\infty}$,

$a b-$ конкатенация слов $a$ и $b$,

$R_{1} R_{2}$ - произведение события $R_{1}$ и события или сверхсобытия $R_{2}$, то есть все слова (сверхслова) вида $a b$, где $a \in R_{1}, b \in R_{2}$,

$R^{*}$ - итерация события $R$, то есть множество $R^{*}=\left\{a_{1} a_{2} a_{3} \ldots a_{k} \mid a_{i} \in R, k \geqslant 1\right\}$,

$R^{\infty}-$ сверхитерация события $R$, то есть

$$
R^{\infty}=\left\{a_{1} a_{2} a_{3} \ldots \mid a_{i} \in R, i=1,2,3, \ldots\right\}
$$

$O(a)-$ множество букв, встречающихся в слове или сверхслове $a$,

$\lim (\alpha)-$ множество букв, встречающихся в сверхслове $\alpha$ бесконечное число раз. 
В статье рассматриваются конечные инициальные автоматы, то есть

$$
\mathfrak{U}=\left(A, Q, B, \varphi, \psi, q_{0}\right),
$$

где $A-$ входной алфавит, $Q-$ множество состояний, которое является конечным подмножеством некоторого фиксированного счетного множества, $B-$ выходной алфавит, $\varphi: A \times Q \rightarrow Q-$ функция переходов, $\psi: A \times Q \rightarrow B-$ функция выходов, $q_{0}$ - начальное состояние.

Если на вход автомату $\mathfrak{U}$ подается сверхслово $\alpha$, на выходе получается сверхслово $y$, и $q_{t}$ означает состояние автомата в момент времени $t$, то функционирование автомата задается системой

$$
\begin{aligned}
y(t) & =\psi\left(\alpha(t), q_{t-1}\right), \\
q_{t} & =\varphi\left(\alpha(t), q_{t-1}\right) .
\end{aligned}
$$

Выходное сверхслово автомата $\mathfrak{U}$ при подаче на его вход сверхслова $\alpha$ будем обозначать $y_{\alpha}^{\mathfrak{A}}$.

Далее в качестве входного алфавита $A$ будет рассматриваться множество $\{0,1\}$. Автомат $\mathfrak{U}$ угадывает сверхслово $\alpha \in\{0,1\}^{\infty}$, если

$$
\sum_{i=1}^{\infty}\left|y_{\alpha}^{\mathfrak{A}}(i)-\alpha(i+1)\right|<\infty \text {. }
$$

Если $\alpha \in\{0,1\}^{\infty}$, то введем обозначение

$$
c^{\mathfrak{A}}(\alpha)=\varliminf_{t \rightarrow \infty} \frac{1}{t} \sum_{i=1}^{t}\left(1-\left|y_{\alpha}^{\mathfrak{A}}(i)-\alpha(i+1)\right|\right) .
$$

Будем говорить, что автомат $\mathfrak{U}$ угадывает (предвосхищает) сверхслово $\alpha$ со степенью $\sigma \in[0,1]$, если $c^{\mathfrak{A}}(\alpha)=\sigma, c^{\mathfrak{A}}(\alpha)$ называется степенью предвосхищения. Автомат $\mathfrak{U}$ угадывает (предвосхищает) некоторое множество сверхслов $S \subset\{0,1\}^{\infty}$ со степенью $\sigma$, если $c^{\mathfrak{Y}}(\alpha) \geqslant \sigma$ для всех $\alpha \in S$.

Считаем, что множество сверхслов частично угадываемо, если существует автомат, такой, что степень предвосхищения для каждого сверхслова множества строго больше нуля.

Определим регулярное событие над алфавитом $A$.

(1) $\{\varnothing\},\{a\}, a \in A,-$ регулярные события.

(2) Пусть $R_{1}, R_{2}$ - регулярные события. Тогда события $R_{1} R_{2}, R_{1} \cup R_{2}$ и $R_{1}^{*}$ также регулярны.

Определим общерегулярное событие над алфавитом $A$.

(1) Если $R$ - регулярное событие над алфавитом $A$, то $R^{*}-$ общерегулярное сверхсобытие над алфавитом $A$.

(2) Если $R_{1}$ - регулярное событие над алфавитом $A, R_{2}$ - общерегулярное сверхсобытие над алфавитом $A$, то $R_{1} R_{2}$ - общерегулярное сверхсобытие над алфавитом $A$.

(3) Если $R_{1}, R_{2}$ - общерегулярные сверхсобытия над алфавитом $A$, то $R_{1} \cup R_{2}-$ общерегулярное сверхсобытие над алфавитом $A$. 
Сверхсобытие $R$ называется представимым в автомате $\mathfrak{U}=\left(A, Q, B, \varphi, \psi, q_{0}\right)$ с помощью семейства подмножеств выходного алфавита $B^{\prime} \subseteq 2^{B}$, если

$$
R=\left\{\alpha \mid \alpha \in A^{\infty}, \lim \left(\psi\left(q_{0}, a\right)\right) \in B^{\prime}\right\}
$$

Будем обозначать $O\left(Q^{\prime}\right)$ множество выходных символов подмножества состояний $Q^{\prime} \subseteq Q$ некоторого конечного автомата $(A, Q, B, \varphi, \psi)$, а именно,

$$
O(Q)=\left\{\psi(q, a) \mid q \in Q^{\prime}, a \in A\right\} .
$$

В любом автомате можно выделить сильно связные компоненты $Q_{1}, \ldots, Q_{k}$, то есть совокупности состояний, достижимых друг из друга: для всех $q^{\prime}, q^{\prime \prime} \in Q_{i}, i=1, \ldots, k$, найдется $\alpha \in\{0,1\}^{*}$ такое, что $\varphi\left(q^{\prime}, \alpha\right)=q^{\prime \prime}$.

Назовем сильно связную компоненту замкнутой, если из нее не достижима никакая другая сильно связная компонента. Очевидно, что хотя бы одна замкнутая сильно связная компонента существует.

Теорема 1. Пусть общерегулярное сверхсобытие $R^{\infty}$ представимо в конечном инициальном автомате $\mathfrak{U}=\left(\{0,1\}, Q, B, \varphi, \psi, q_{0}\right),|Q|=n$, с помощью некоторого $B^{\prime} \subset 2^{B}$, тогда следующие утверждения эквивалентны.

(1) Сверхсобытие $R^{\infty}, R \subseteq\{0,1\}^{*}$, не является частично угадываемым;

(2) Для всех $\alpha \in\{0,1\}^{*}$ найдутся $\beta \in\{0,1\}^{*}, \gamma \in\{0,1\}^{\infty}$ такие, что $\beta \alpha \gamma \in R^{\infty}$;

(3) Хотя бы для одной замкнутой сильно связной компоненты $\widetilde{Q}$ автомата $\mathfrak{U}$ справедливо включение $O(\widetilde{Q}) \in B^{\prime}$.

Теорема 2. Существвует алгоритм, определяющий, является ли сверхсобытие $R^{\infty}$, представимое в приведенном автомате с $n$ состояниями, частично угадываемыл, и если ответ положителен, строящий автомат с частичным предвосхищением, причем сложность алгоритма линейно зависит от $n$.

Теорема 3. Общерегулярное сверхсобытие $R_{1}^{\prime} R_{1}^{\infty} \cup R_{2}^{\prime} R_{2}^{\infty} \cup \ldots \cup R_{k}^{\prime} R_{k}^{\infty}$, где $R_{1}, \ldots, R_{k}$, $R_{1}^{\prime}, \ldots, R_{k}^{\prime}$ - некоторые регулярные события, частично угадываемо тогда и только тогда, когда частично угадываемо каждое из сверхсобытий $R_{1}^{\infty}, \ldots, R_{k}^{\infty}$.

Теорема 4. Сущуествует алгоритм, определяющий, является ли сверхсобытие, представимое в приведенном автомате с $n$ состояниями, частично угадываемым, и его сложность линейно зависит от $n$. Так же существует алгоритм построения автомата $c$ частичным предвосхищением в случае, если сверхсобытие частично угадываемо.

\section{3. Критерий частичного предвосхищения сверхитерации события}

Пусть множество замкнутых сильно связных компонент автомата $\mathfrak{U}$ есть $\widetilde{Q}_{1}, \ldots, \widetilde{Q}_{w}$, $w \geqslant 1$. Рассмотрим два случая. 
I. В автомате найдется замкнутая сильно связная компонента $\widetilde{Q}^{\prime}$, такая, что множество букв, выдаваемое этими состояниями, есть элемент $B^{\prime}$, то есть существуют $i \in\{1, \ldots, w\}$, $\tilde{b} \in B^{\prime}$ такие, что

$$
\left\{\psi(q, a) \mid a \in A, q \in \widetilde{Q}_{i}\right\}=\tilde{b} .
$$

Докажем, что в этом случае

(a) $R^{\infty}$ не является частично угадываемым;

(б) для всех $\alpha \in\{0,1\}^{*}$ найдутся $\beta \in\{0,1\}^{*}, \gamma \in\{0,1\}^{\infty}$ такие, что $\beta \alpha \gamma \in R^{\infty}$;

II. Такой замкнутой сильно связной компоненты не найдется. Докажем, что в этом случае

(в) множество является частично угадываемым;

(г) найдется слово $\alpha \in\{0,1\}^{*}$, которое не является подсловом никакого сверхслова из $R^{\infty}$.

Из утверждений (а)-(г), очевидно, следует утверждение теоремы 1.

Докажем утверждение (а). Для произвольного автомата

$$
\mathfrak{B}=\left(\{0,1\}, S,\{0,1\}, \varphi^{\prime}, \psi^{\prime}, q_{0}\right)
$$

построим сверхслово $\alpha \in R^{\infty}$, для которого $c^{\mathfrak{B}}(\alpha)=0$.

Замкнутая сильно связная компонента $\widetilde{Q}^{\prime}$, множество выходных букв состояний которой есть $\tilde{b} \in B^{\prime}$, достижима из начального состояния автомата $\mathfrak{U}$ по некоторому слову $\beta \in\{0,1\}^{*}: \varphi\left(q_{0}, \beta\right)=q^{\prime} \in \widetilde{Q}^{\prime}$, где $\beta-$ префикс $\alpha \in\{0,1\}^{\infty}$, подается на вход автомата B. Далее сверхслово строится так:

$$
\alpha(t+1)=\overline{\psi^{\prime}\left(q_{0},\left.\alpha\right|_{t}\right)} .
$$

Это сверхслово не будет угадано автоматом $\mathfrak{B}$ ни с какой ненулевой степенью.

Возможны два случая.

1. Построенное таким образом $\alpha \in R^{\infty}$, то есть $\lim (\psi(\alpha)) \in B^{\prime}$.

2. $\alpha \notin R^{\infty}$. Из предположения I следует, что существуют состояния $q_{1}^{\prime}, q_{2}^{\prime}, \ldots, q_{m}^{\prime}$ и набор букв $a_{1}, a_{2}, \ldots, a_{m}, a_{i} \in\{0,1\}, i=1, \ldots, m$, что $\psi\left(q_{i}^{\prime}, a_{i}\right)=b_{i}, i=1, \ldots, m$, где $\left\{b_{1}, \ldots, b_{m}\right\}=\tilde{b} \in B^{\prime}$. Для любого состояния $q_{j} \in \tilde{Q}^{\prime}$ найдется такое слово $\gamma_{j}$ длины, меньшей $n m<n^{2}$, что множество букв слова $\bar{\psi}\left(q_{j}, \gamma_{j}\right)$ есть $\tilde{b}$.

Построим нужное сверхслово следующим образом:

$$
\tilde{\alpha}=\beta \alpha_{1} \gamma_{j_{1}} \alpha_{2} \gamma_{j_{2}} \ldots \alpha_{i} \gamma_{j_{i}} \ldots,
$$

где $\alpha_{i}$ - полностью неугадываемое слово для состояния $\varphi\left(q_{0}, \beta \alpha_{1} \gamma_{j_{1}} \ldots \alpha_{i-1} \gamma_{j_{i-1}}\right)$ автомата $\mathfrak{B}$ длины $i n^{2}$, а слово $\gamma_{j_{i}}$ соответствует состоянию $q_{j_{i}}=\varphi\left(q_{0}, \beta \alpha_{1} \gamma_{j_{1}} \ldots \alpha_{i}\right)$.

Подсчитаем степень предвосхищения:

$$
\begin{aligned}
c^{\mathfrak{B}}(\tilde{\alpha}) & \leqslant \lim _{t \rightarrow \infty} \frac{\sum_{i=1}^{t}\left|\gamma_{j_{i}}\right|}{\sum_{i=1}^{t}\left|\gamma_{j_{i}}\right|+\sum_{i=1}^{t}\left|\alpha_{i}\right|+|\beta|} \\
& \leqslant \lim _{t \rightarrow \infty} \frac{t n^{2}}{n^{2}(1+2+\ldots+t)+t n^{2}} \\
& =\lim _{t \rightarrow \infty} \frac{t}{t(t+1) / 2+t}=\lim _{t \rightarrow \infty} \frac{2}{t+3}=0 .
\end{aligned}
$$


Докажем утверждение (б). Для произвольного $\alpha$ найдем такие $\beta \in\{0,1\}^{*}, \gamma \in\{0,1\}^{\infty}$, что $\beta \alpha \gamma \in R^{\infty}$. Рассмотрим замкнутую сильно связную компоненту $\widetilde{Q}^{\prime}$. Она достижима из начального состояния по некоторому слову $\beta \in\{0,1\}^{*}: \varphi\left(q_{0}, \beta\right)=q^{\prime} \in \widetilde{Q}^{\prime}$. Для $q^{\prime}$, как и для любого состояния этой компоненты, справедливо равенство $\varphi\left(q^{\prime}, \alpha\right)=$ $q^{\prime \prime} \in \tilde{Q}^{\prime}$. Построим $\gamma$ как последовательность $\gamma_{i_{t}}$ из п. (а), где $\gamma_{i_{t+1}}$ соответствует $q_{i_{t+1}}=\varphi\left(\beta \alpha \gamma_{i_{1}} \ldots \gamma_{i_{t}}\right), t=0,1,2, \ldots$, а $\gamma_{i_{1}}$ строится для $q_{i_{1}}=\varphi\left(q_{0}, \beta \alpha\right)$. По построению, $\lim (\psi(\beta \alpha \gamma)) \in B^{\prime}$ и, следовательно, $\beta \alpha \gamma \in R^{\infty}$.

Докажем утверждение (в). Построим автомат $\mathfrak{B}$ с частичным предвосхищением данного множества. Для этого оставим структуру автомата $\mathfrak{U}$, в котором представимо $R^{\infty}$, и зададим выходные функции. Согласно условию II, для любой замкнутой сильно связной компоненты $\widetilde{Q}_{i}$ выполнено соотношение $O\left(\widetilde{Q}_{i}\right) \notin B^{\prime}$. Покажем, что тогда любое сверхслово, приводящее автомат из начального состояния в эту компоненту, не будет принадлежать $R^{\infty}$.

Предположим, что найдется сверхслово $\lambda \in R^{\infty}$, переводящее автомат в некоторую замкнутую сильно связную компоненту $\widetilde{Q}$, то есть в компоненте $\widetilde{Q}$ найдутся состояния $q_{1}, \ldots, q_{m}$ и буквы $a_{1}, \ldots, a_{m}$, такие, что $\psi\left(q_{i}, a_{i}\right)=b_{i}, i=1, \ldots, m$, где $\left\{b_{1}, \ldots, b_{m}\right\}=$ $b \in B^{\prime} \subset 2^{B}$. Также состояния данной компоненты могут выводить некоторые буквы $c_{1}, \ldots, c_{k}$, такие, что $\left\{b_{1}, \ldots, b_{m}, c_{1}, \ldots, c_{k}\right\} \notin B^{\prime}$. Для каждого $c_{i}, i=1, \ldots, k$, найдутся (необязательно единственные) $q_{i}^{\prime} \in \widetilde{Q}$ и буква $a_{i}^{\prime}$ такие, что $\psi\left(q_{i}^{\prime}, a_{i}^{\prime}\right)=c_{i}$.

Найдется такое натуральное число $t^{*}$, что $\varphi\left(q_{0}, \operatorname{pref}\left(\lambda, t^{*}\right)\right)=q_{1}$.

Каждое состояние в этой компоненте достижимо из каждого. Поэтому можно построить такое слово $\theta^{1}$ длины, не большей $n^{2}$, что

$$
O\left(\psi\left(q_{1}, \theta^{1}\right)\right)=\left\{b_{1}, \ldots, b_{m}, c_{1}, \ldots, c_{k}\right\} .
$$

Например, найдутся $t_{1} \in\left\{0<t_{2}<\ldots<t_{m}<t_{1}^{\prime}<t_{2}^{\prime}<\ldots<t_{k}^{\prime}\right\}$ такие, что

$$
\begin{aligned}
\varphi\left(q_{1}, \operatorname{pref}\left(\theta^{1}, t_{i}\right)\right) & =q_{i}, \quad i=1, \ldots, m, \\
\varphi\left(q_{1}, \operatorname{pref}\left(\theta^{1}, t_{i}^{\prime}\right)\right) & =q_{i}^{\prime}, \quad i=1, \ldots, k, \\
\theta^{1}\left(t_{i}+1\right) & =a_{i}, \\
\theta^{1}\left(t_{i}^{\prime}+1\right) & =a_{i}^{\prime},
\end{aligned}
$$

и $\varphi\left(q_{1}, \theta^{1}\right)=q_{1}$.

Ясно, что сверхслово $\operatorname{pref}\left(\lambda, t^{*}\right) \theta^{1} \operatorname{post}\left(\lambda, t^{*}\right) \in R^{\infty}$, так как

$$
\lim \left(\bar{\psi}\left(q_{0}, \operatorname{pref}\left(\lambda, t^{*}\right) \theta^{1} \operatorname{post}\left(\lambda, t^{*}\right)\right)=\lim \left(\bar{\psi}\left(q_{0}, \lambda\right)\right) \in B^{\prime}\right) .
$$

Тогда его можно представить как $r_{1} r_{2} \ldots r_{i} \ldots$, где $r_{i} \in R, i=1,2, \ldots$ Возьмем наименьшее $p^{1}$ такое, что $\left|r_{1} \ldots r_{p^{1}}\right| \geqslant t^{*}+\left|\theta^{1}\right|$. Введем обозначение $q^{1}=\varphi\left(q_{0}, r_{1} \ldots r_{p^{1}}\right)$. Далее, подадим слово $\operatorname{pref}\left(\lambda, t^{*}\right)$ на состояние $q^{1}$ и построим слово $\theta^{2}$ такое, что

$$
O\left(\bar{\psi}\left(q^{1}, \operatorname{pref}\left(\lambda, t^{*}\right) \theta^{2}\right)\right)=\left\{b_{1}, \ldots, b_{m}, c_{1}, \ldots, c_{k}\right\},
$$

и при этом

$$
\varphi\left(q_{0}, \operatorname{pref}\left(\lambda, t^{*}\right) \theta^{2}\right)=q_{1} .
$$

Так же, как и длина $\theta^{1}$, длина $\theta^{2}$ не превосходит $n^{2}$.

Далее, верхслово $\operatorname{pref}\left(\lambda, t^{*}\right) \theta^{2} \operatorname{post}\left(\lambda, t^{*}\right) \in R^{\infty}$. Следовательно, также в $R^{\infty}$ попадает сверхслово $r_{1} \ldots r_{p^{1}} \operatorname{pref}\left(\lambda, t^{*}\right) \theta^{2} \operatorname{post}\left(\lambda, t^{*}\right)$, то есть

$$
r_{1} \ldots r_{p^{1}} \operatorname{pref}\left(\lambda, t^{*}\right) \theta^{2} \operatorname{post}\left(\lambda, t^{*}\right)=r_{1} \ldots r_{p^{1}} r_{p^{1}+1} \ldots \in R^{\infty}, \quad r_{i} \in R .
$$


Тогда выберем наименьшее число $p^{2}$, такое что $p^{2} \geqslant t^{*}+\left|\theta^{2}\right|$, и введем обозначение

$$
q^{2}=\varphi\left(q^{1}, r_{p^{1}+1} \ldots r_{p^{2}}\right)
$$

Таким образом, можно построить сверхслово

$$
\begin{aligned}
\operatorname{pref}\left(\lambda, t^{*}\right) \theta^{1} \operatorname{pref}\left(\lambda, t^{*}\right) \theta^{2} & \ldots \operatorname{pref}\left(\lambda, t^{*}\right) \theta^{n+1} \operatorname{post}\left(\lambda, t^{*}\right) \\
& =r_{1} \ldots r_{p^{n+1}} \operatorname{post}\left(\lambda, t^{*}\right) \in R^{\infty}, \quad r_{i} \in R, \quad i=1, \ldots, p^{n+1},
\end{aligned}
$$

такое, что

$$
O\left(\bar{\psi}\left(q_{i}, \operatorname{pref}\left(\lambda, t^{*}\right) \theta^{i+1}\right)\right)=\left\{b_{1}, \ldots, b_{m}, c_{1}, \ldots, c_{k}\right\}, \quad i=1, \ldots, n+1,
$$

но

$$
\lim \left(\bar{\psi}\left(r_{1} \ldots r_{p^{n+1}} \operatorname{post}\left(\lambda, t^{*}\right)\right)\right)=\left\{b_{1}, \ldots, b_{m}\right\}
$$

При этом

$$
q^{i}=\varphi\left(q_{0}, r_{1} \ldots r_{p^{i}}\right), \quad i=1, \ldots, n+1 .
$$

Очевидно, найдутся такие номера $i$ и $j, i<j \leqslant n+1$, что $q_{i}$ и $q_{j}$ обозначают одно и то же состояние. Тогда сверхслово

$$
\rho=\operatorname{pref}\left(\lambda, t^{*}\right) r_{1} \ldots r_{p^{i}}\left(r_{p^{i}+1} \ldots r_{p^{j}}\right)^{\infty} \in R^{\infty}
$$

по построению. Следовательно, оно должно приниматься автоматом $\mathfrak{A}$. Но

$$
\lim \left(\bar{\psi}\left(q_{0}, \rho\right)\right)=\left\{b_{1}, \ldots, b_{m}, c_{1}, \ldots, c_{k}\right\} \notin B^{\prime} .
$$

Получено противоречие с предположением, что существует замкнутая сильно связная компонента $\widetilde{Q}$ такая, что $O(\tilde{Q}) \supset \tilde{b} \in B^{\prime}$. Тем самым, $O\left(\widetilde{Q}^{\prime}\right) \nsupseteq \tilde{b}$ для всех $\tilde{b} \in B^{\prime}$ и для любой замкнутой сильно связной компоненты $\tilde{Q}^{\prime}$. Таким образом, в случае II все замкнутые сильно связные компоненты тупиковые, то есть сверхслово из $R^{\infty}$ из начального состояния в них не попадает.

Разобьем множество состояний автомата на тупиковые и нетупиковые:

$$
\begin{aligned}
& Q=T \cup N \\
& T=\left\{q \mid q \in \widetilde{Q}_{i}, i=1, \ldots, w\right\} .
\end{aligned}
$$

Выходные функции определим как функции от состояний, в которые приходит автомат, то есть

$$
\psi^{\prime}(q(t), x(t))=f(q(t+1))=f(\varphi(q(t), x(t))) .
$$

Пусть

$$
\mathfrak{B}=\left(\{0,1\}, Q,\{0,1\}, \varphi, \psi^{\prime}\right)
$$

есть искомый автомат. Функцию выхода в тупиковых состояниях определим произвольным образом, а выходные функции остальных состояний устроены так, чтобы по выбранной букве автомат попадал в состояние, наиболее далекое от тупикового. 

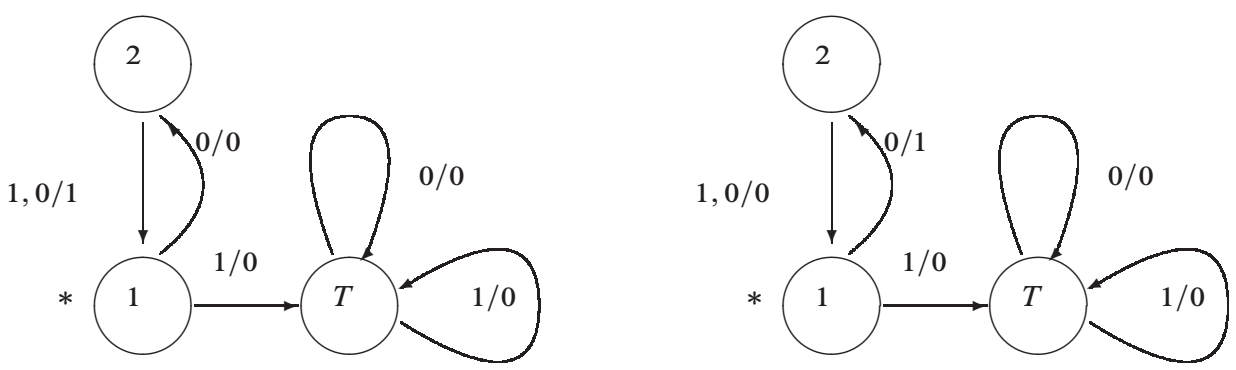

Рис. 1. Автомат, в котором сверхсобытие $(01 \cup 00)^{\infty}$ представимо с помощью $\{\{0,1\},\{1\}\}$, и автомат, частично угадывающий это сверхсобытие

1-й шаг. Выделим состояния, из которых тупиковое состояние достижимо по одному ребру. Тогда если $\varphi(q, x) \in T$, то $f(q)=\bar{x}$. Класс выделенных состояний обозначим

$$
Q(1)=\{q \notin T \mid \exists x: \varphi(q, x) \in T\} .
$$

$i$-й шаг. Пусть выделены классы $Q(1), \ldots, Q(i-1)$, и для этих состояний выходные функции определены. Рассмотрим множество

$$
Q(i)=\{q \in N /\{Q(1) \cup \ldots \cup Q(i-1)\} \mid \exists x \in\{0,1\}: \varphi(q, x) \in Q(i-1)\} .
$$

Зададим выходные функции следующим образом: если для $q \in Q(i), x \in\{0,1\}$ справедливо включение $\varphi(q, x) \in Q(i-1)$, то

$$
f(q)=\bar{x} .
$$

Очевидно, что не позднее, чем через $n-1$ шаг функция выхода будет определена.

Покажем, что в любом сверхслове исходного множества данный автомат не может не угадывать $n-1$ символ подряд. Действительно,

$$
N=\bigcup_{i=1}^{n^{\prime}} Q(i), \quad n^{\prime}<n .
$$

В состояниях $Q(1)$ на сверхсловах из $R^{\infty}$ автомат всегда угадывает. В остальных нетупиковых состояниях по построению автомат $\mathfrak{B}$ либо угадывает символ $\bar{x}$, либо переходит из $Q(i)$ в $Q(i-1)$. Таким образом, автомат угадывает множество со степенью, не меньшей, чем $1 /(n-1)$. Заметим, что в случае $n=1$ частичного угадывания не может быть.

Наконец, докажем утверждение (г). Построим слово $\alpha$, по которому из любого состояния $\mathfrak{U}$ достижимы состояния $T$, что будет означать, что это слово не является подсловом никакого сверхслова из $R^{\infty}$. 
Пусть

$$
N=\left\{q_{1}, \ldots, q_{|N|}\right\} .
$$

Известно, что для всех $q_{i}, i=1, \ldots,|N|$ существует $\alpha_{i}$ такое, что $\varphi\left(q_{i}, \alpha_{i}\right) \in T$, так как все замкнутые сильно связные компоненты являются тупиковыми. Можно также заметить, что суммарная длина $\alpha_{i}$ не превосходит $(|N|(|N|-1)) / 2$.

Будем строить слово индуктивно.

Шаг 0: $i_{1}=1$. Пусть $\alpha^{1}=\alpha_{i_{1}}$. Если $\alpha^{1}$ не является подсловом никакого сверхслова из $R^{\infty}$, то слово $\alpha=\alpha^{1}$ построено, в противном случае переходим к следующему шагу.

Шаг 1. Из предположения предыдущего пункта следует, что найдутся $\beta_{1} \in\{0,1\}^{*}$, $\gamma_{1} \in\{0,1\}^{\infty}$ такие, что $\beta_{1} \alpha^{1} \gamma_{1} \in R^{\infty}$.

Пусть $\varphi\left(q_{0}, \beta_{1}\right)=q_{i^{\prime}}$, причем $q_{i^{\prime}} \neq i_{1}$, поскольку $\varphi\left(q_{i_{1}}, \alpha^{1}\right) \in T$.

Пусть теперь $i_{2}=i^{\prime}$, а $\alpha^{2}=\alpha^{1} \alpha_{i_{2}}$. Если $\alpha^{2}$ не является подсловом никакого сверхслова из $R^{\infty}$, то построение закончено, в противном случае переходим к следующему шагу.

Шаг $j$. Пусть уже построено слово $\alpha^{j}=\alpha_{i_{1}} \alpha_{i_{2}} \ldots \alpha_{i_{j}}$, являющееся подсловом некоторого сверхслова из $R^{\infty}$, и $i_{k} \neq i_{l}$ при $k \neq l, k, l=1, \ldots, j$. Существуют такие $\beta_{j} \in\{0,1\}^{*}, \gamma_{j} \in\{0,1\}^{\infty}$, что $\beta_{j} \alpha^{j} \gamma_{j} \in R^{\infty}$.

Рассмотрим $q_{i^{\prime}}=\varphi\left(q_{0}, \gamma_{j}\right)$. Известно, что $i^{\prime} \notin\left\{i_{1}, \ldots, i_{j}\right\}$, так как $\varphi\left(q_{i_{k}}, \alpha^{k}\right) \in T$, $k=1, \ldots, j$.

Назначим $i_{j+1}=i^{\prime}$, а $\alpha^{j+1}=\alpha^{j} \alpha_{i_{j+1}}$. Переход к следующему шагу осуществляется в случае, если $\alpha^{j+1}$ является подсловом некоторого сверхслова из $R^{\infty}$.

Если слова $\alpha^{1}, \alpha^{2}, \ldots, \alpha^{n-2}$ были подсловами сверхслов данного множества, то слово $\alpha^{n-1}$ уже таковым не будет, потому что, поданное на любое состояние из $N$ автомата $\mathfrak{A}$, оно переводит его в некоторое состояние из $T$.

Теорема 1 доказана.

В доказательстве теоремы 1 содержится идея, как по автомату $\mathfrak{A}$, в котором представима сверхитерация регулярного события, определить, возможно ли частичное предвосхищение.

Определение частичной угадываемости состоит в выделении сильно связных компонент автомата, для чего существуют алгоритмы, работающие линейное время (например, алгоритм на с. 214 в [4]), и в сравнении выходов замкнутых сильно связных компонент $O(\widetilde{Q})$ с элементами множества $B^{\prime}$, что тоже занимает линейное время (меньшее, чем $\left.n\left|B^{\prime}\right|^{2}\right)$.

В случае, если множество является частично угадываемым, можно также построить угадывающий его автомат. Если автомат $\mathfrak{U}$ задан диаграммой Мура, то построение новых выходных функций (пункт $c$ ) доказательства) занимает время $n$, а переход от другого способа задания автомата к диаграмме Мура осуществляется за линейное время. Таким образом, доказана и теорема 2. 


\section{4. Критерий предвосхищения общерегулярных сверхсобытий}

Из утверждения теоремы 1 также вытекает другой способ частичного угадывания. Пусть известно, что $R^{\infty}$ не содержит некоторого подслова $a_{1} \ldots a_{l}$ (длины $l \leqslant n(n-1) / 2$ ). Тогда можно построить автомат со структурой задержки на $l-1$ такт, но с тем отличием, что при поступлении на вход $a_{1}, a_{2}, \ldots, a_{l-1}$ автомат по букве $a_{l}$ переходит в тупиковое состояние, а не в $a_{2} \ldots a_{l}$, а выходные функции устроены так же, как в доказательстве теоремы (п. (в)).

Рассмотрим автомат

$$
\circlearrowleft\left(a_{1} \ldots a_{l}\right)=\left(\{0,1\}, Q,\{0,1\}, \varphi, g, q_{0}\right),
$$

где

$$
Q=\left\{q_{T}\right\} \cup\{a(1) \ldots a(l-1) \mid a(i) \in\{0,1\}, i=1, \ldots, l-1\}
$$

and

$$
\begin{gathered}
\varphi(a(1) \ldots a(l-1), x)=a(2) \ldots a(l-1) x \quad \text { если } a(1) \ldots a(l-1) \neq a_{1} \ldots a_{l-1}, \\
\varphi\left(a_{1} \ldots a_{l-1}, \bar{a}_{l}\right)=a_{2} \ldots \bar{a}_{l}, \quad \varphi\left(a_{1} \ldots a_{l-1}, a_{l}\right)=q_{T}, \quad \varphi\left(q_{T}, x\right)=q_{T}, \\
g\left(q_{T}\right)=0, \quad g\left(a_{1} \ldots a_{l-1}\right)=\bar{a}_{l} .
\end{gathered}
$$

Для каждого $a(1) \ldots a(l-1)$ найдем наибольшее $k$ такое, что

$$
a(1) \ldots a(l-1)=a(1) \ldots a(l-k-1) a_{1} \ldots a_{k} .
$$

Тогда

$$
g\left(a(1) \ldots a_{k}\right)=\bar{a}_{k+1} .
$$

В случае, если наибольшее $k=0, g(a(1) \ldots a(l-1))=\bar{a}_{1}$. Это состояние объявляется начальным $q_{0}$.

В автомате такого вида состояние $a_{1} \ldots a_{l-1}$ достижимо из любого другого, кроме тупикового, по пути длины, не большей $l-1$. Поэтому не может быть более $l$ неугаданных символов подряд, следовательно, степень угадывания не меньше $1 /(l-1)$.

В случае $l>n$ такой алгоритм невыгоден, но он удобен для случая произвольных общерегулярных множеств.

Перейдем к доказательству теоремы 3.

Если хотя бы одно $R_{i}^{\infty}$ не является частично угадываемым, то уже есть подмножество данного множества сверхслов $R_{i}^{\prime} R_{i}^{\infty}$, которое ни один автомат не сможет частично угадать.

Пусть теперь все $R_{i}^{\infty}$ частично угадываемы, и следовательно, каждое из них содержит все подслова. Построим автомат, частично угадывающий это множество.

Для каждого $R_{i}^{\infty}$ возьмем подслово $a^{i}$ наименьшей длины $l_{i}$, не встречающееся в соответствующих сверхсловах. Может быть два и более таких слов одинаковой длины, но произвольным образом выбирается одно. Упорядочим их произвольным образом и получим $a^{1}, \ldots, a^{k}$.

Для каждого $a^{i}$ длины $l_{i}$ строится инициальный автомат

$$
\circlearrowleft\left(a^{i}\right)=\left(\{0,1\}, Q^{i},\{0,1\}, \varphi^{i}, g^{i}, q_{0}^{i}\right),
$$




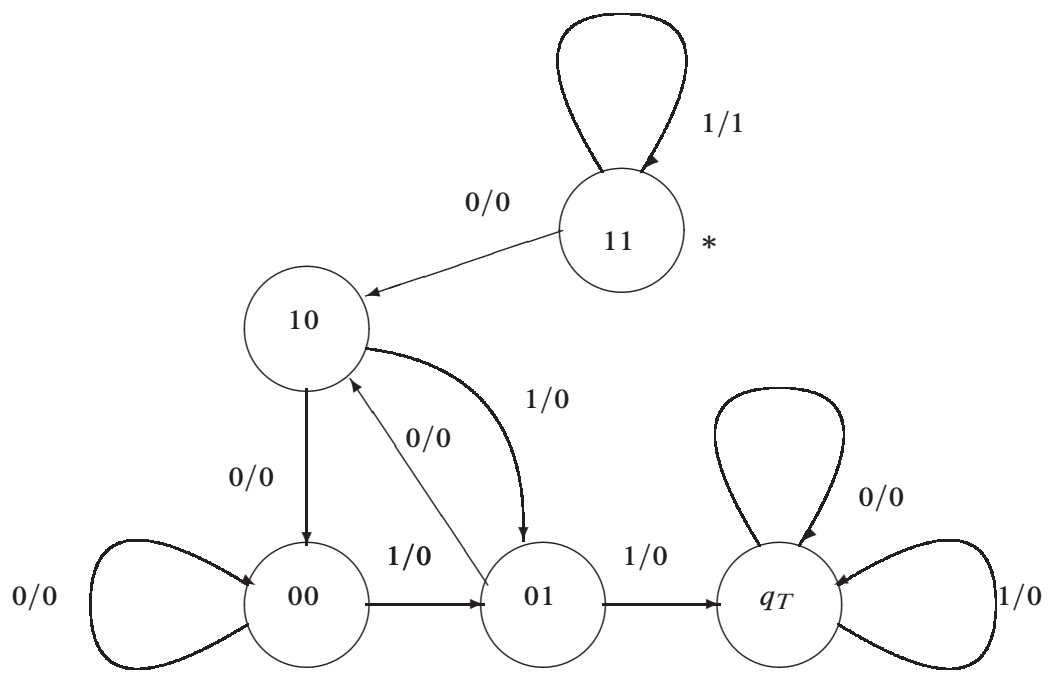

Рис. 2. Автомат $\mathscr{G}(011)$

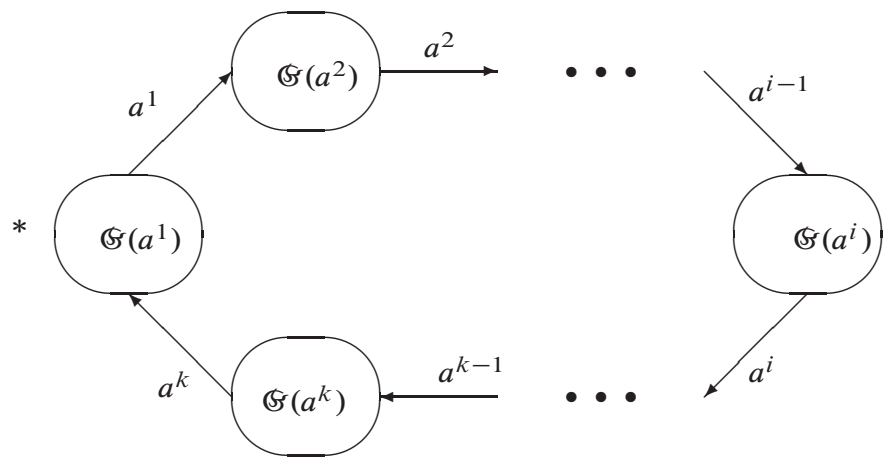

Рис. 3. Автомат $\mathfrak{B}$

построение которого описано выше. Далее автоматы соединяются в один

$$
\mathfrak{B}=\left(\{0,1\}, Q^{1} \cup \ldots \cup Q^{k} /\left\{q_{T}^{1} \cup \ldots \cup q_{T}^{k}\right\},\{0,1\}, \varphi, g, q_{0}^{1}\right)
$$

следующим образом: на состояниях $Q^{i} /\left\{q_{T}^{i}, a^{i}\right\}$ выполняются соотношения $\varphi \equiv \varphi^{i}$ и $\left.\varphi\left(a_{i}\right]_{l_{i}-1}, a_{i}\left(l_{i}\right)\right)=q_{0}^{i+1}$, то есть если встречается комбинация букв, которая не должна встречаться на предполагаемом множестве, осуществляется переход к автомату для другого множества, в его начальное состояние. В состояниях последнего автомата $\$\left(a^{k}\right)$ переход снова к первому $\left(5\left(a^{1}\right)\right.$.

Рассмотрим работу такого автомата на сверхслове $\alpha \in R_{j}^{\prime} R_{j}^{\infty} \subset R_{1}^{\prime} R_{1}^{\infty} \cup \ldots \cup R_{n}^{\prime} R_{k}^{\infty}$. Каждое такое $\alpha$ можно представить в виде $\beta \gamma, \beta \in R_{j}^{\prime}, \gamma \in R_{j}^{\infty}$. При подаче слова $\beta$ возможны встречи как подслова $a^{j}$, так и других. Через конечное время $|\beta|$ сверхслово точно не будет содержать в себе комбинацию букв $a^{j}$. Если автомат находится в состоя- 
ниях $\varsigma^{j}\left(a^{j}\right)$, то, во-первых, он из них не выйдет по этому сверхслову $\gamma$, а во-вторых, он будет предсказывать его со степенью, не меньшей $1 /\left(l_{j}-1\right)$.

Если же автомат находится в состояниях $Q^{t}$, то до тех пор, пока в сверхслове $\gamma$ не встретится комбинация букв $a^{t}$ (а она может и не встретиться, и тогда степень угадывания будет не меньше $\left.1 /\left(l_{t}-1\right)\right)$, автомат будет находиться там. Если поступит $a^{t}$, то автомат перейдет в состояния $Q^{t+1}$ и так далее. В итоге автомат окажется в $Q^{j}$, либо в состояниях $Q^{t^{\prime}}$, если подслово $a^{t^{\prime}}$ в $\alpha$ также не встречается.

Таким образом, любое сверхслово $\alpha$ из данного множества будет угадано со степенью

$$
c^{\mathfrak{B}}(\alpha) \geqslant \frac{1}{\max _{i}\left(l_{i}\right)-1}
$$

Теорема 3 доказана.

Утверждение теоремы 4 о существовании алгоритма, устанавливающего возможность частичного предвосхищения и работающего линейное время, есть следствие теорем 2 и 3 . Построение соответствующего автомата описано в доказательстве теоремы 3.

\section{Список литературы}

1. Трахтенброт Б. А., Бардзинь Я. М., Конечные автоматы (поведение и синтез). Наука, Москва, 1970.

2. Вереникин А. Г., Гасанов Э. Э., Об автоматной детерминизации множеств сверхслов. Дискретная математика (2006) 18, №2, 84-97.

3. Мастихина А. А., О частичном угадывании сверхслов. Интеллектуальные системы (2007) 11, 609-619.

4. Sedgewick R., Algorithms in C++. Part 5: Graph algorithms. Addison-Wesley, Reading, Mass., 2001.

5. Кудрявцев В. Б., Алешин С. В., Подколзин А. С., Введение в теорию автоматов. Наука, Москва, 1985.

Статья поступила 1.09. 2010. 